\title{
Analysis of Mismatched First Order A Priori Information in Iterative Source-Channel Decoding
}

\begin{abstract}
B. Schotsch, P. Vary, T. Clevorn
Due to complexity and delay constraints a usually significant amount of residual redundancy remains in the source samples after source coding. This residual redundancy can be exploited by iterative source-channel decoding for error concealment and quality improvements. One key design issue in joint source-channel (de-)coding is the index assignment. Besides conventional index assignments optimized index assignments have been developed, e.g., considering zeroth or first order a priori information of the source samples. However, in real-world scenarios it is unlikely that the amount of residual redundancy is constant over time and thus it may occur that the just deployed index assignment is suboptimal at times when the residual redundancy differs too much from the amount that it is optimized for. In this paper the performance of optimized index assignments is examined that consider first order a priori knowledge under such suboptimal conditions.
\end{abstract}

Keywords: iterative source-channel decoding, optimized index assignments, first order a priori knowledge, EXIT charts

\section{Introduction}

Since the discovery of Turbo codes [1], which allow for channel coding close to the Shannon limit with moderate complexity, the Turbo principle of exchanging extrinsic information has been extended to various components of the receiver chain. Iterative source-channel decoding (ISCD) [2, $3]$ is such an extension. Instead of a concatenation of two or more channel decoders, a channel decoder and a soft decision source decoder (SDSD) $[4,5]$ are iteratively combined exchanging extrinsic information. Unlike Turbo channel decoding, which aims at minimizing the bit error rate, ISCD mainly aims at error concealment and signal restoration which is not necessarily connected to a lower bit error rate, but to a higher parameter SNR. ISCD exploits the a priori knowledge on the residual redundancy of the source codec parameters that remains after imperfect source coding. The a priori knowledge can be a nonuniform probability distribution, an autocorrelation or a cross correlation. The source codec parameters can be, e.g., scale factors or predictor coefficients for speech, audio and video signals. Delay or complexity constraints prevent a complete removal of the residual redundancy and therefore, in practice, a quite large amount of residual redundancy remains in the source codec parameters, which can be exploited by ISCD.

One major design issue in ISCD systems is the index assignment. Besides the traditional index assignments for noniterative systems, such as natural binary or Gray, optimized index assignments have been developed that take into account the possible feedback due to the Turbo loop between channel decoder and SDSD. In [6, 7] index assignments have been introduced that are optimized considering a nonuniform probability distribution, i.e., the zeroth order a priori information, of source samples. Further enhanced index assignments have been presented in [8] and the corresponding optimization process even takes the first order a priori information, e.g., the autocorrelation, of the source samples into account. This paper focuses on the latter type of index assignments.

In general, an index assignment is chosen in advance and is not exchanged during a transmission, otherwise side infor- mation has to be transmitted to notify the receiver of the change. However, in this paper the index assignment is assumed to be constant during a transmission. If it is a priori known that the source codec parameters bear a specific overall autocorrelation, an appropriate index assignment can be applied exploiting this autocorrelation. But since most signals have a time-varying autocorrelation it has to be examined how much the performance degrades, if the signal correlation does not match the correlation that the index assignment is optimized for.

At first, the underlying ISCD transmission scheme is described in Section 2. Since in this paper the performance of index assignments that consider first order a priori knowledge will be under examination, more details on this topic will be given in Section 3. In Section 4 the performance (degradation) of the ISCD system is shown by means of simulation results utilizing the index assignments under both optimal and suboptimal conditions. In Section 5 the simulated scenarios are then analyzed and confirmed by so-called EXIT (extrinsic information transfer) charts [9].

\section{Iterative source-channel decoding}

The baseband model of the utilized ISCD transmission scheme is depicted in Fig. 1. Source codec parameters $u$ are generated by a Gauss-Markov source, with an inherent autocorrelation $\rho$ in order to obtain comparable and reproducible results. At time instant $\tau, K$ source codec parameters $u_{k, \tau}$ are assigned to one frame $\underline{u}_{\tau}$ with $k=0,1, \ldots, K-1$ denoting the position in the frame. In this paper the autocorrelation $\rho$ is constant in order to simulate a fixed mismatch between the correlation $\rho_{\mathrm{T}}$ of the transmitted source parameters and the assumed correlation $\rho_{\mathrm{R}}$ at the receiver. The autocorrelation takes on values from a finite set, e.g., $\rho \in\{0.0,0.1, \ldots, 0.9\}$. The value-continuous and time-discrete source samples $u_{k, \tau}$ are each quantized to a quantizer reproduction level $\bar{u}_{k, \tau} \in U$, where $U$ is the quantizer codebook. To each $\bar{u}_{k, \tau}$ a unique bit 


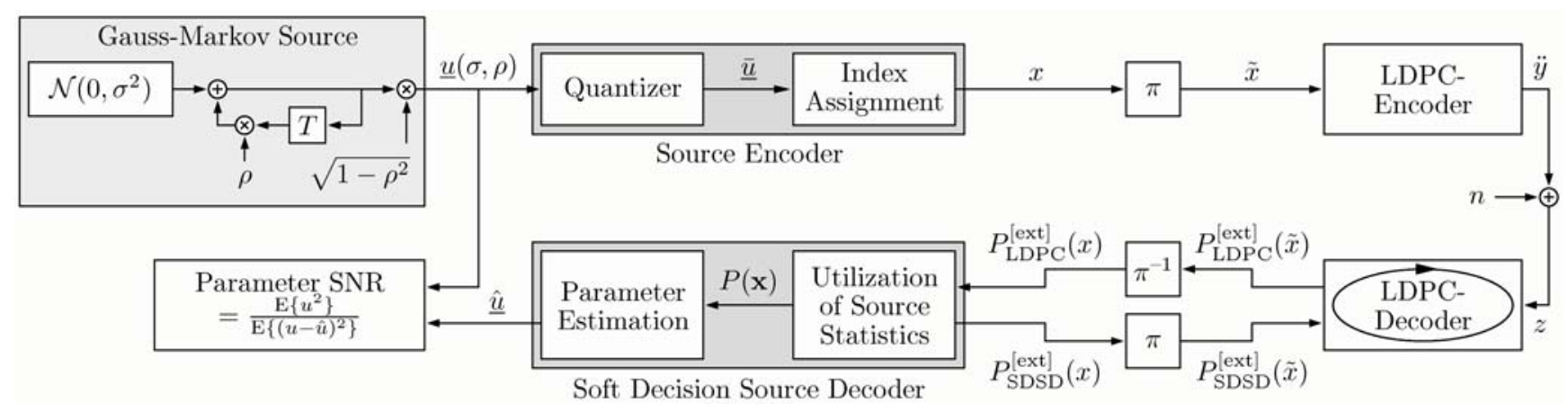

(SDSD)

Fig. 1: Baseband model of ISCD with LDPC codes

pattern $\mathbf{x}_{k, \tau}$ of $M$ bits is assigned according to the utilized index assignment. The single bits of a bit pattern $\mathbf{x}_{k, \tau}$ are indicated by $x_{k, \tau}^{(m)}$ with $m=0,1, \ldots, M-1$, and the frame of bit patterns is denoted as $\underline{\mathbf{x}}_{\tau}$. Three parameter SNR optimized index assignments considering first order a priori knowledge (SOAK1) are used and the natural binary (NB) index assignment serves as a reference. The SOAK1 index assignments are optimized for different source correlations, thus they are referred to as $\operatorname{SOAK}(\rho)$.

The bit interleaver $\pi$ scrambles the incoming bits $x$ of the frame $\underline{\mathbf{x}}_{\tau}$ of bit patterns to $\widetilde{x}$ in a deterministic manner. To simplify the notation, we restrict the interleaving to a single time frame with index $\tau$ and omit the time frame index $\tau$ in the following, where appropriate.

For the channel encoding of a frame $\underline{\mathbf{x}}$ of interleaved bits $x$ we utilize LDPC codes, which were first proposed by Gallager [10] and rediscovered by MacKay [11]. LDPC codes have a very high error correction capability with iterative decoding that is very close to the Shannon limit. Their performance is comparable or even superior to that of convolutional Turbo codes. In this paper we use a modification of short LDPC codes as presented in [12]. Identical instances of a short LDPC code are combined to a long LDPC code, whose frame size is flexible in multiples of a subframe size, i.e., the frame size of the short LDPC code. By serially concatenating the subframes with a bit-interleaver and a second component that provides extrinsic information according to the Turbo principle (e.g., a soft decision source decoder (SDSD) as in this paper), extrinsic information can also be exchanged between subframes. Such concatenated LDPC codes approach very well the performance of long monolithic LDPC codes of the same frame size [12]. The performance of the concatenated LDPC code strongly depends on the performance of the short code. Therefore, the short code has to be chosen carefully. As short LDPC code a $(21,11)$ difference set cyclic (DSC) code [13] is used. DSC codes feature a high minimum Hamming distance, and especially at short block lengths they can outperform comparable pseudo-random LDPC codes [14].

The resulting codeword is denoted as $\mathbf{y}$ with bits $y$, which are mapped to bipolar bits $\ddot{y} \in\{ \pm 1\}$ for BPSK transmission with symbol energy $E_{s}=1$. We choose the simple BPSK modulation scheme, since modulation is no design issue in this paper.

On the channel, the signal $\ddot{y}$ is superposed with additive white Gaussian noise (AWGN) $n$ with the known power spectral density $s_{n}^{2}=N_{0} / 2$, i.e., $z=\ddot{y}+n$. The received symbols $z$ are evaluated in a Turbo process, in which extrinsic reliabilities between the LDPC decoder and the SDSD are exchanged. Utilizing LDPC codes results in an additional iterative loop in the LDPC decoder, in which extrinsic information is exchanged between the variable nodes and the check nodes. These iterations are denoted as LDPC-iterations.

Details about the ISCD receiver can be found in [2, 3, 15]. The LDPC decoder uses the belief propagation algorithm $[16,11]$ to generate extrinsic information. The SDSD determines the extrinsic information mainly from the natural residual source redundancy, which generally remains in the bit patterns $\mathbf{x}_{k}$ after source encoding. Such residual redundancy appears on the parameter level, e.g., as a nonuniform distribution $P\left(\underline{u}_{k}\right)$, in terms of a correlation, or as other possible time-dependencies. The latter terms of residual redundancy are generally approximated by a first order Markov chain, i.e., by exploiting the conditional probabilities $P\left(\mathbf{x}_{k} \mid \mathbf{x}_{k-1}\right)$. These conditional probabilities heavily depend on the source correlation. For specific source correlations, e.g., $\rho \in\{0.0,0.1$, $\ldots, 0.9\}$, they can be calculated once in advance. The technique for combining this a priori information $P\left(\mathbf{x}_{k} \mid \mathbf{x}_{k-1}\right)$ on the parameter level with the soft input values $P_{\mathrm{LDPC}}^{[\mathrm{ext}]}(x)$ on the bit level is also well known in the literature. The algorithm for the computation of the extrinsic $P_{\mathrm{LDPC}}^{[\mathrm{ext}]}(x)$ has been detailed, e.g., 
in $[2,3,15]$. As a quality measure we consider the parameter signal-to-noise ratio (SNR)

$$
P=10 \log _{10} \frac{E\left\{|u|^{2}\right\}}{E\left\{|u-\hat{u}|^{2}\right\}}
$$

\section{Index assignments}

The index assignment is a major design factor influencing the performance of ISCD transmission systems. Several conventional index assignments already exist, such as natural binary (NB) or Gray [17]. They are well-suited for noniterative systems $[15,7,6]$, but they exhibit only a suboptimal performance in ISCD.

In this paper we consider only bit patterns consisting of $M=3$ bit that are assigned to $Q=2^{M}=8$ quantizer levels. The utilized index assignments are listed in Table 1, but first, the optimization algorithm will be briefly explained. This was introduced in [8] and can be found in more detail there. According to the notation in [15], the index assignment $\Gamma: \bar{u} \mapsto \mathbf{x}$ is given in the corresponding decimal representation $\{\mathbf{x}\}_{10}$ for an increasing quantizer level $\bar{u}$. The quantizer levels are consecutively numbered, i.e., $\bar{u}^{(0)}, \bar{u}^{(1)}, \ldots, \bar{u}^{(7)}$ for $Q=8$. Thus, the decimal notation of the index assignment, e.g., SOAK1 for $\rho=0.9$ is (cf. Table 1)

$$
\begin{aligned}
\bar{u}^{(0)} & \mapsto\left(\{\mathbf{x}\}_{10}=0 \hat{=}\{\mathbf{x}\}_{2}=000\right) \\
\bar{u}^{(1)} & \mapsto\left(\{\mathbf{x}\}_{10}=6 \hat{=}\{\mathbf{x}\}_{2}=110\right) \\
\vdots & \\
\bar{u}^{(7)} & \mapsto\left(\{\mathbf{x}\}_{10}=7 \hat{=}\{\mathbf{x}\}_{2}=111\right) .
\end{aligned}
$$

Parameter SNR optimized index assignments that take into account first order a priori knowledge are generated by minimizing the overall noise energy function [8]

$D^{[\text {SOAK } 1]}=\frac{1}{M Q} \sum_{\bar{u}_{\tau} \in U} \sum_{m=1}^{M} \sum_{\bar{u}_{\tau-1} \in U} P\left(\overline{\bar{u}}_{\tau}, \bar{u}_{\tau}, \bar{u}_{\tau-1}\right)\left|\bar{u}_{\tau}-\overline{\bar{u}}_{\tau}\right|^{2} \rightarrow$ min,

with $P\left(\overline{\bar{u}}_{\tau}, \bar{u}_{\tau}, \bar{u}_{\tau-1}\right)=P\left(\bar{u}_{\tau} \mid \bar{u}_{\tau-1}\right) P\left(\bar{u}_{\tau} \mid \bar{u}_{\tau-1}\right) P\left(\bar{u}_{\tau-1}\right)$. The term $\left|\bar{u}_{\tau}-\overline{\bar{u}}_{\tau}\right|^{2}$ corresponds to the noise energy that originates from estimating the quantizer level $\breve{\bar{u}}_{\tau}$ instead of the correct quantizer level $\bar{u}_{\tau}$ at time instant $\tau . \breve{u}_{\tau}$ corresponds to the bit pattern that differs from the bit pattern of $\bar{u}_{\tau}$ only at position m. Both $\bar{u}_{\tau}$ and $\bar{u}_{\tau}$ are assumed to have the same predecessor $\bar{u}_{\tau-1}$. Also only single bit errors are taken into account, since the system is generally supposed to operate in good channels, in which the probability of two or more bit errors occurring in one bit pattern is negligible. In order to determine the overall noise energy, each term $\left|\bar{u}_{\tau}-\overline{\bar{u}}_{\tau}\right|^{2}$ has to be weighted by the probability of its occurrence $P\left(\overline{\bar{u}}_{\tau}, \bar{u}_{\tau}, \bar{u}_{\tau-1}\right)$ and has to be summed up. Finally, the noise energy function has to be minimized either by an exhaustive search for small values of $M$ $(M \leq 4)$, which yields a global optimum, or by the binary switching algorithm $[18,6,15]$, which may lead to a local optimum only.

Table 1: Index assignments $\Gamma: \bar{u} \mapsto \mathbf{x}$ from $Q=8$ quantizer levels $\bar{u}=0, \ldots, Q-1$, to bit patterns $\mathbf{x}$ with $M=3$ bit

\begin{tabular}{|lr|c|}
\hline Index Assignment $\Gamma$ & $\{\mathbf{x}\}_{10}=\Gamma(\bar{u})$ \\
\hline natural binary (NB) & & $0,1,2,3,4,5,6,7$ \\
\hline & $\rho=0.0$ & $0,1,3,2,7,6,4,5$ \\
$\begin{array}{l}\text { parameter SNR optimized } \\
\text { (SOAK1) }\end{array}$ & $\rho=0.7$ & $0,1,3,2,6,4,5,7$ \\
\cline { 3 - 3 } & $\rho=0.9$ & $0,6,5,1,3,2,4,7$ \\
\hline
\end{tabular}

\section{Simulation Results}

In Fig. 2 the parameter SNR performance of ISCD utilizing the optimized index assignments is compared to the one using the conventional natural binary index assignment. The parameter SNR performance is shown for various combinations of the source parameter correlation at the transmitter $\rho_{\mathrm{T}}$ and the assumed correlation at the receiver $\rho_{\mathrm{R}}$. For exploiting the residual redundancy at the receiver it is necessary that the source parameter correlation is known at the receiver. To that end, the source correlation either has to be transmitted as side information or it has to be estimated at the receiver. The latter approach has turned out to be very precise and easy to implement. However, in this paper, the mismatch between $\rho_{\mathrm{T}}$ and $\rho_{\mathrm{R}}$ is preset.

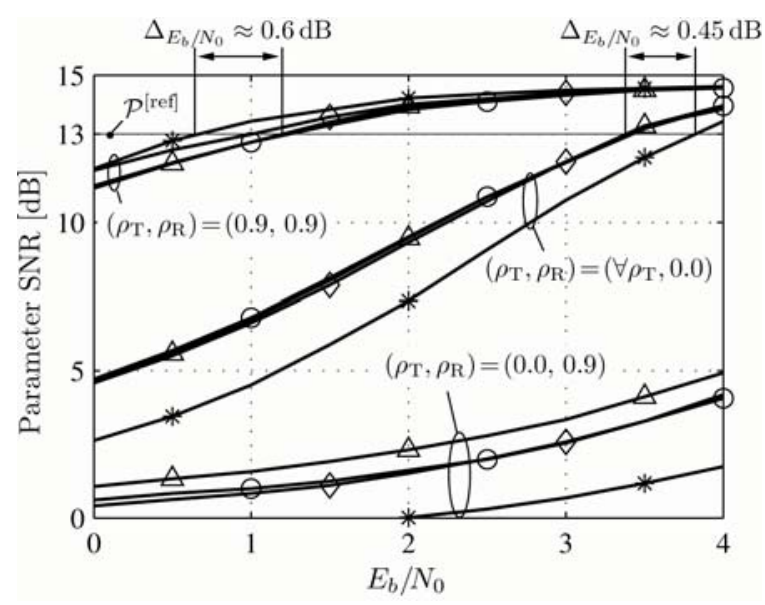

Frame size $K=330,8$-level LMQ, $\left(\mathrm{L}^{3} \mathrm{~S}\right)^{6}$ iterations

$$
\begin{array}{ll}
\rightarrow \operatorname{SOAK} 1(\rho=0.9) & \checkmark \operatorname{SOAK} 1(\rho=0.7) \\
\bigcirc \operatorname{SOAK} 1(\rho=0.0) & \triangle \mathrm{NB} \text { (reference) }
\end{array}
$$

Fig. 2: Parameter SNR performance of source correlation optimized index assignments $\operatorname{SOAKl}_{3}^{8}(\rho)$ in combination with an autocorrelation mismatch 
A reference parameter SNR of $P^{[\text {ref }]}=13 \mathrm{~dB}$ is assumed at which the parameter SNR performances will be compared. The parameters emitted by the Gauss-Markov source $\left(\sigma^{2}=1\right)$ exhibiting a source correlation of $\rho_{\mathrm{T}}$, are grouped to frames of size $K=330$ and are quantized by an 8-level Lloyd-Max quantizer (LMQ) resulting in 990 uncoded bits per frame. After channel encoding this yields 1890 coded bits per frame. On the receiver side, $\left(\mathrm{L}^{3} \mathrm{~S}\right)^{6}$ iterations are performed, which means that during each of the six iterations between LDPC-decoder and SDSD three LDPC-iterations between the variable nodes and the check nodes of the LDPC-decoder are carried out.

The set of curves in Fig. 2 that is labeled by

$$
\left(\rho_{\mathrm{T}}, \rho_{\mathrm{R}}\right)=\left(\forall \rho_{\mathrm{T}}, 0.0\right)
$$

shows the scenario in which no correlation is exploited at the receiver, independently of the actual source correlation. This is also the current state of today's transmission systems, where the available correlation is not utilized at the receiver in order to enhance the signal quality. In this case the three curves for $\operatorname{NB}, \operatorname{SOAK} 1(\rho=0.0)$ and $\operatorname{SOAK} 1(\rho=0.7)$ show about the same performance, while for $\operatorname{SOAK} 1(\rho=0.9)$ a degradation of $\Delta_{E_{b} / N_{0}} \approx 0.6 \mathrm{~dB}$ can be observed.

The leftmost set of curves shows the case in which $\rho_{\mathrm{R}}=\rho_{\mathrm{T}}=0.9$ are matching. Such high values for $\rho_{\mathrm{T}}$ are not unusual for several source codec parameters in current communication systems like GSM or UMTS. The parameter SNR optimized index assignment $\operatorname{SOAK} 1(\rho=0.9)$ yields the highest gain of $\Delta_{E_{b} / N_{0}} \approx 0.6 \mathrm{~dB}$ compared to the reference index assignment NB . The gain of $\operatorname{SOAK} 1(\rho=0.7)$ is already negligibly small and the performances of $\operatorname{SOAK} 1(\rho=0.0)$ and NB are almost the same. However, this shows that if the source parameters exhibit a certain amount of correlation it is definitely expedient to exploit it.

The rightmost set of curves labeled by $\left(\rho_{\mathrm{T}}, \rho_{\mathrm{R}}\right)=(0.0,0.9)$ displays the performance of the scenario in which the source parameters are uncorrelated, but the receiver assumes a high correlation $\left(\rho_{\mathrm{R}}=0.9\right)$. When $\rho_{\mathrm{R}} \gg \rho_{\mathrm{T}}$ a high performance degradation occurs for all index assignments, but for NB the least and for $\operatorname{SOAK} 1(\rho=0.9)$ the highest degradation can be observed. However, this scenario is very unlikely, since the correlation can be estimated very accurately at the receiver, so that this high mismatch is temporally limited to the instances of abrupt changes of the source parameter correlation.

In systems with a high and slowly varying source correlation a reliable estimation of the source correlation is possible, and thereby it is reasonable and feasible to exploit the performance gain of an index assignment optimized for high source correlations. In systems with fast and high source correlation fluctuations a more conservative choice of the index assignment is recommended.

\section{EXIT chart analysis}

In this section the system is analyzed by EXIT (extrinsic information transfer) charts [9], which are a powerful tool for analyzing and optimizing the convergence behavior of iterative systems utilizing the Turbo principle, i.e., systems exchanging and refining extrinsic information. The capabilities of the components, in our case the LDPC decoder and the soft decision source decoder (SDSD), are analyzed separately. The extrinsic mutual information $I^{[\mathrm{ext}]}$ obtained by each component is determined for a certain a priori mutual information $I^{[\text {apri] }}$. Both, $I^{[\mathrm{ext}]}$ and $I^{[\mathrm{apri}]}$, are calculated on the basis of the actual data and the available extrinsic or a priori information of the data. As a basis for this calculation usually histograms of the respective L-values, e.g., $L_{\mathrm{LDPC}}^{[\mathrm{ext}]}$ for $L_{\mathrm{LDPC}}^{[\text {ext }]}$, are used. L-values are the log-likelihood ratios of the corresponding probabilities $P$ [19]. For the EXIT characteristics, the a priori L-values are simulated as uncorrelated Gaussian distributed random variables with variance $\sigma_{A}^{2}$ and mean $\mu=\sigma_{A}^{2} / 2$. In most cases, e.g., for a classical Turbo Code [1], this is a good assumption [9]. The applicability of EXIT charts to ISCD is demonstrated, e.g., in [15].

Since the extrinsic information of one component serves as input a priori information for the other component, the two resulting EXIT characteristics are plotted in a single graph with swapped axes. The EXIT characteristic of the LDPC decoder depends on the $E_{b} / N_{0}$ of the channel, while the EXIT characteristic of the SDSD is independent of the $E_{b} / N_{0}$ since the SDSD has no access to the received channel symbols $z$. The decoding trajectory (step curve) shows the mutual information $I$ for each iteration in a simulation of the complete system. In the optimization process of the system design the components are chosen such that the (first) intersection of their EXIT characteristics moves towards the point $\left(L_{\mathrm{LDPC}}^{[\mathrm{ext}]}, L_{\mathrm{SDSD}}^{[\mathrm{ext}]}\right)=(1,1)$. In the design of the system, i.e., the design of the characteristics, we have always $\rho_{\mathrm{T}}=\rho_{\mathrm{R}}$, while in the actual simulation settings $\rho_{\mathrm{T}}=\rho_{\mathrm{R}}$ is not necessarily fulfilled. Thus, when the system design matches the actual settings, the decoding trajectory approaches this intersection, indicating that the best possible performance is obtained. The number of steps of the decoding trajectory corresponds to the number of useful iterations.

Fig. 3(a) depicts the EXIT charts corresponding to the results with the $\operatorname{SOAK} 1(\rho=0.9)$ index assignment in Fig. 2 at 


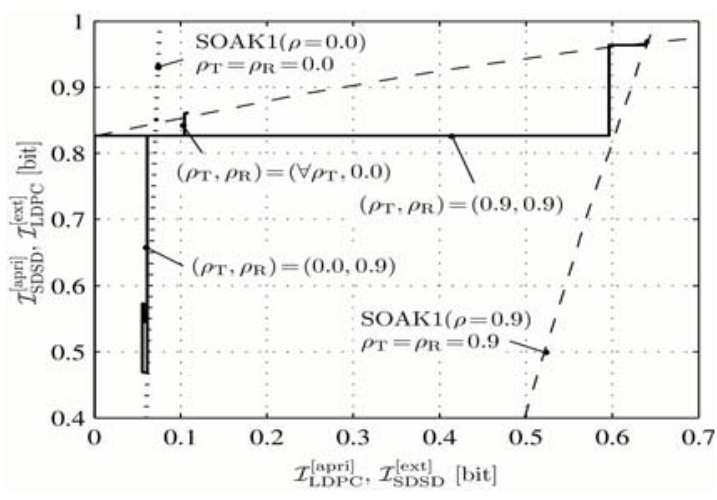

a) EXIT trajektories for SOAK1 $(\rho=0.9)$

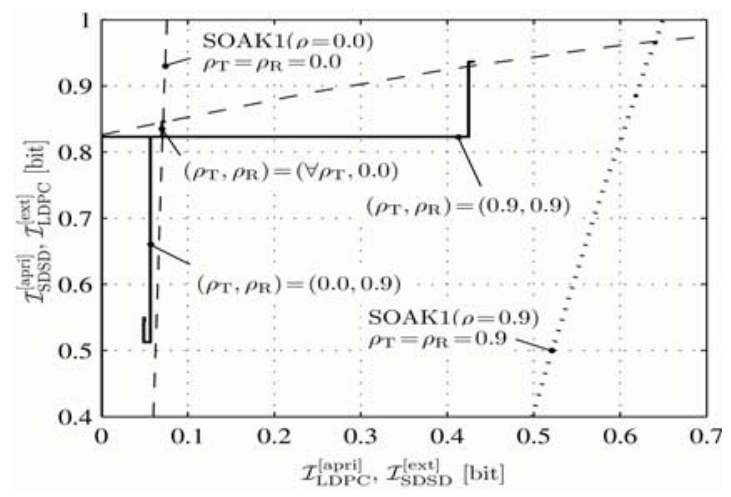

b) EXIT trajektories for SOAK1 $(\rho=0.0)$

Fig. 3: EXIT charts for $\operatorname{SOAK} 1(\rho=0.0)$ and $\operatorname{SOAK} 1(\rho=0.9)$

$E_{b} / N_{0}=2 \mathrm{~dB}$. We can observe that when the system design with the index assignment $\operatorname{SOAK} 1(\rho=0.9)$ matches the simulated settings with $\left(\rho_{\mathrm{T}}, \rho_{\mathrm{R}}\right)=(0.9,0.9)$, the decoding trajectory reaches the intersection of the LDPC EXIT characteristic and the $\operatorname{SOAK} 1(\rho=0.9)$ EXIT characteristic, confirming the excellent performance of this case in Fig. 2. In the other cases the decoding trajectory ends very early at low amounts of mutual information, especially at low values of $I_{\text {SDSD }}^{[\mathrm{ext}]}$. Here, only values in the range of the $\operatorname{SOAK} 1(\rho=0.0)$ EXIT characteristic are obtained. With $\left(\rho_{\mathrm{T}}, \rho_{\mathrm{R}}\right)=(0.0,0.9)$ even a significant decrease in mutual information occurs with an increasing number of iterations. This yields the poor results of this case in Fig. 2.

Fig. 3(b) depicts the EXIT charts of the results with the $\operatorname{SOAK} 1(\rho=0.0)$ index assignment in Fig. 2, again at $E_{b} / N_{0}=2 \mathrm{~dB}$. As expected, with the simulation settings matching the system design, i.e., $\left(\rho_{\mathrm{T}}, \rho_{\mathrm{R}}\right)=(0.0,0.0)$ in this case, we reach the intersection of the $\operatorname{SOAK} 1(\rho=0.0) \operatorname{EXIT}$ characteristic with the LDPC EXIT characteristic. However, this intersection occurs at much lower values of mutual information than the intersection with the $\operatorname{SOAK1}(\rho=0.9)$ EXIT characteristic, which is relevant in Fig. 3(a). This relation can also be observed in the corresponding parameter SNR performance in Fig. 2. The two cases with a mismatch differ noticeably in the $\operatorname{SOAK} 1(\rho=0.0)$ EXIT chart. For $\left(\rho_{\mathrm{T}}, \rho_{\mathrm{R}}\right)=(0.0,0.9)$, which corresponds to a significant overestimation at the receiver, we get a decreasing decoding trajectory, quite similar to the respective case in Fig. 2. But when $\left(\rho_{\mathrm{T}}, \rho_{\mathrm{R}}\right)=(0.9,0.9)$, the ISCD receiver correctly uses the high residual redundancy due to the high autocorrelation and significantly outperforms the $\left(\rho_{\mathrm{T}}, \rho_{\mathrm{R}}\right)=(0.0,0.0)$ case. Only the wrong index assignment $\operatorname{SOAK} 1(\rho=0.0)$ instead of $\operatorname{SOAK} 1(\rho=0.9)$ lets the $\left(\rho_{\mathrm{T}}, \rho_{\mathrm{R}}\right)=(0.9,0.9)$ decoding trajectory fall short of the $\operatorname{SOAK} 1(\rho=0.9)$ EXIT characteristic.
Taking Fig. 3(a) and Fig. 3(b) into account, we can observe that by means of EXIT charts we can accurately analyze, explain and confirm the parameter SNR results.

\section{Conclusion}

In this paper the performance of iterative source-channel decoding utilizing optimized index assignments has been analyzed. The studied index assignments are optimized with respect to the parameter SNR and by considering first order a priori knowledge. The simulation results show that high gains are achievable if the source parameter correlation is high and if the correlation at the receiver matches. These findings have been confirmed by an EXIT chart analysis. With optimized index assignments additional gains can be achieved compared to conventional index assignments. In case the assumed correlation at the receiver is higher than the source parameter correlation, high deteriorations can occur depending on how mismatched the correlations are. Thus, depending on the dynamics and the amount of correlation of the source codec parameters, either the optimized or the conventional index assignments are better suited. The index assignments optimized for high correlations perform better in systems with a high and slowly varying source correlation, while the conventional index assignments have an advantage in systems with fast and high source correlation fluctuations.

\section{References}

[1] Berrou, C., Glavieux, A., Thitimajshima, P.: Near Shannon Limit Error-Correcting Coding and Decoding, in IEEE International Conference on Communications (ICC), Geneva, Switzerland, May 1993.

[2] Adrat, M., Vary, P., Spittka, J.: Iterative Source-Channel Decoder Using Extrinsic Information from Softbit-Source Decoding, in IEEE International Conference on Acoustics, Speech, and Signal Processing (ICASSP), Salt Lake City, UT, USA, May 2001.

[3] Görtz, N.: On the Iterative Approximation of Optimal Joint Source-Channel Decoding, IEEE Journal on Selected Areas in Communications, Sept. 2001, p. 1662-1670. 
[4] Fingscheidt, T., VARY P.: Softbit Speech Decoding: A New Approach to Error Concealment, IEEE Transactions on Speech Audio Processing, Mar. 2001, p. 240-251.

[5] Vary, P., Martin R.: Digital Speech Transmission: Enhancement, Coding and Error Concealment, John Wiley \& Sons Ltd, 2006.

[6] Görtz, N.: Optimization of Bit Mappings for Iterative Source-Channel Decoding, in $3^{\text {rd }}$ International Symposium on Turbo Codes $\mathcal{E}^{2}$ Related Topics, Brest, France, Sept. 2003.

[7] Hagenauer, J., Görtz, N.: The Turbo Principle in Joint Source-Channel Coding, in IEEE Information Theory Workshop (ITW), Paris, France, Apr. 2003.

[8] Clevorn, T., Vary, P., Adrat, M.: Parameter SNR Optimized Index Assignments and Quantizers based on First Order A Priori Knowledge for Iterative Source-Channel Decoding, in Conference on Information Sciences and Systems (CISS), Princeton, NJ, USA, Mar. 2006.

[9] Ten Brink, S.: Convergence Behavior of Iteratively Decoded Parallel Concatenated Codes, IEEE Transactions on Communications, Oct. 2001, p. 1727-1737.

[10] Gallager, R. G.: Low-Density Parity-Check Codes, IRE Transactions on Information Theory, Jan. 1962, p. 21-28.

[11] Mackay, D. J. C., Neal, R. M.: Near Shannon Limit Performance of Low Density Parity Check Codes, IEE Electronics Letters, Aug. 1996, p. 1645-1646.

[12] Clevorn, T., Oldewurtel, F., Vary, P.: Combined Iterative Demodulation and Decoding using very short LDPC Codes and Rate-1 Convolutional Codes, in Conference on Information Sciences and Systems (CISS), Baltimore, MD, USA, Mar. 2005.

[13] Weldon, E. J.: Difference-set cyclic codes, The Bell Systems Technical Journal, Sept. 1966, p. 1045-1055.

[14] Lucas, R., Fossorier, M., Kou, Y., Lin, S.: Iterative Decoding of One-Step Majority Logic Decodable Codes
Based on Belief Propagation, IEEE Transactions on Communications, June 2000, pp. 931-937.

[15] Adrat, M., Vary, P.: Iterative Source-Channel Decoding: Improved System Design Using EXIT Charts, EURASIP Journal on Applied Signal Processing (Special Issue: Turbo Processing), May 2005.

[16] Pearl, J.: Probabilistic Reasoning in Intelligent Systems: Networks of Plausible Inference, Morgan Kaufmann, 1988.

[17] Gray, F.: Pulse Code Communication, United States Patent Office, US patent 2,632,058, Mar. 1953.

[18] Zeger, K., Gersho, A.: Pseudo-Gray Coding, IEEE Transactions on Communications, Dec. 1990, p. 2147-2158.

[19] Hagenauer, J., Offer, E., Papke, L.: Iterative Decoding of Binary Block and Convolutional Codes, IEEE Transactions on Information Theory, Mar. 1996, p. 429-445.

\section{Birgit Schotsch}

e-mail: schotsch@ind.rwth-aachen.de

Peter Vary

e-mail: vary@ind.rwth-aachen.de

Institute of Communication Systems and Data Processing

RWTH Aachen University

Templergraben 55

52056 Aachen, Germany

Thorsten Clevorn

e-mail: thorsten.clevorn@infineon.com

\section{COM Development Center NRW}

Infineon Technologies AG

Düsseldorfer Landstrasse 401

47259 Duisburg, Germany 